\title{
Testing Fundamental Assumptions: 3-D X-ray Spectrum Imaging of Concrete
}

\author{
Bryan Sudbrink ${ }^{1,2}$, Jeffrey M. Davis ${ }^{1}$, M. Tyler Ley ${ }^{2}$, Keana Scott $^{1}$ \\ 1) National Institute of Standards and Technology, Gaithersburg, MD, 20899-8371 \\ 2) Department of Civil Engineering, Oklahoma State University, Stillwater, OK, 74078
}

Two fundamental assumptions in surface analysis are that the surface is representative of the whole and the analysis area is homogeneous at sampling depth [1]. As surface analysis techniques become more common for analysis of concrete specimens, it is important to test the validity of these assumptions. These assumptions were tested using a method developed to track and summarize the change in composition with depth through analyses with the micro X-ray fluorescence $(\mu \mathrm{XRF})$ instrument.

Typically, the $\mu \mathrm{XRF}$ beam size is $30 \mu \mathrm{m}$ laterally (for laboratory based instruments), and the depth of production for the fluoresced x-rays is approximately $30 \mu \mathrm{m}$ in concrete [2]. After an initial surface analysis in the $\mu \mathrm{XRF}$, a concrete specimen surface was ground using three grades of silicon carbide to remove $30 \mu \mathrm{m}$ of material. Then, another X-ray spectrum image was taken of the same area. The grinding and re-scanning process was repeated 26 times until a total of $780 \mu \mathrm{m}$ of the material were analyzed.

The data from each scan were used to develop compositional similarity models for cement and concrete analysis. The model categorizes the surface of the concrete into areas of similar composition, based on the intensity of each element embedded in the pixels of the $\mu$ XRF image (see Figure 2). A total of seven phases were identified, three of which defined the cement paste based on the varying levels of sulfur in the cement, and four defined the coarse aggregate based on the varying levels of potassium, calcium, and silicon. For each X-ray spectrum image, these seven categories were color coded into an image, as shown in Figure 3. An analysis of relative phase abundances indicates that changes in the aggregate phases occur gradually with depth, whereas alteration in the cement phases can occur immediately.

A three dimensional (depth into the material along the $\mathrm{Z}$ axis) image of the concrete sample was produced from the derived compositional images. Analysis of the images indicates that random surface selection (where the sample is exposed via successive grinding and polishing routines) and analysis of a concrete sample will show a generally representative phase distribution. However, researchers should interrogate samples at other depths in order to ensure a truly representative sample.

\section{References}

[1] Janssens, K. (2000)a. Comparison with Other Microanalytical Techniques. Janssens, K; Adams, F; Rindby, A, Microscopic X-ray Fluorescence Analysis (pp. 215-218).

[2] Janssens, K. (2000)b. Comparison with Other Microanalytical Techniques. In Janssens, K; Adams, F; Rindby, A, Microscopic X-ray Fluorescence Analysis (pp. 229). 


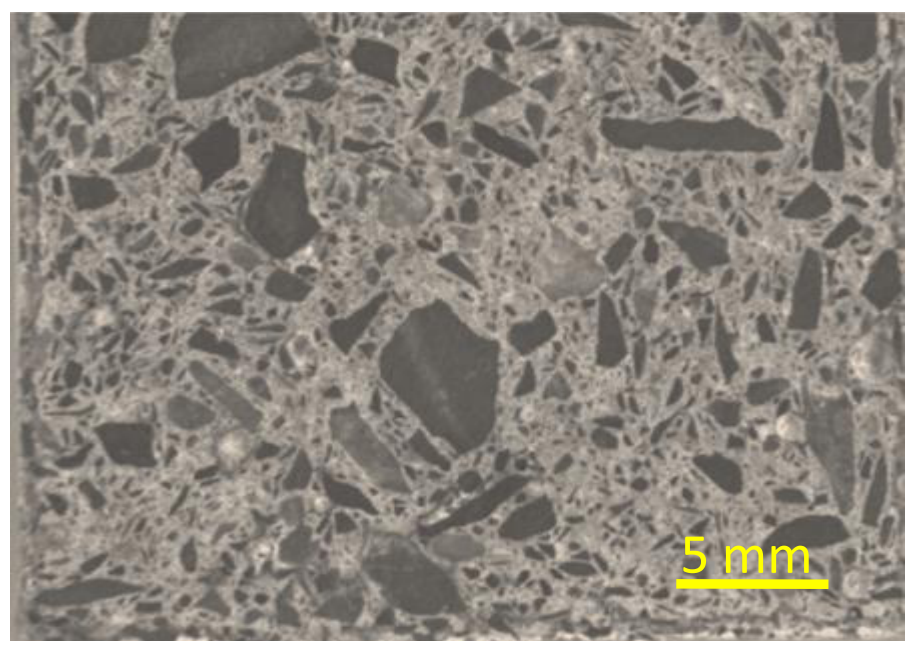

Figure 1: Optical gray scale image of concrete sample. Each time the sample was ground, an optical image was taken of the newly exposed surface

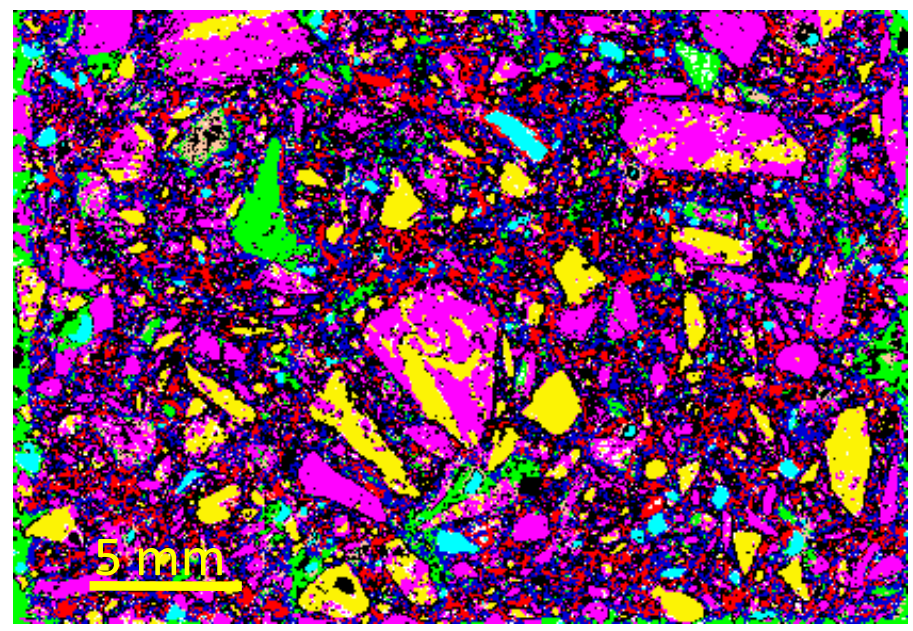

Figure 3: Derived "phase" map. Phases (derived through compositional simility) were assigned to color values throughout the experiment. The phase map was used to summarize the compositional data.

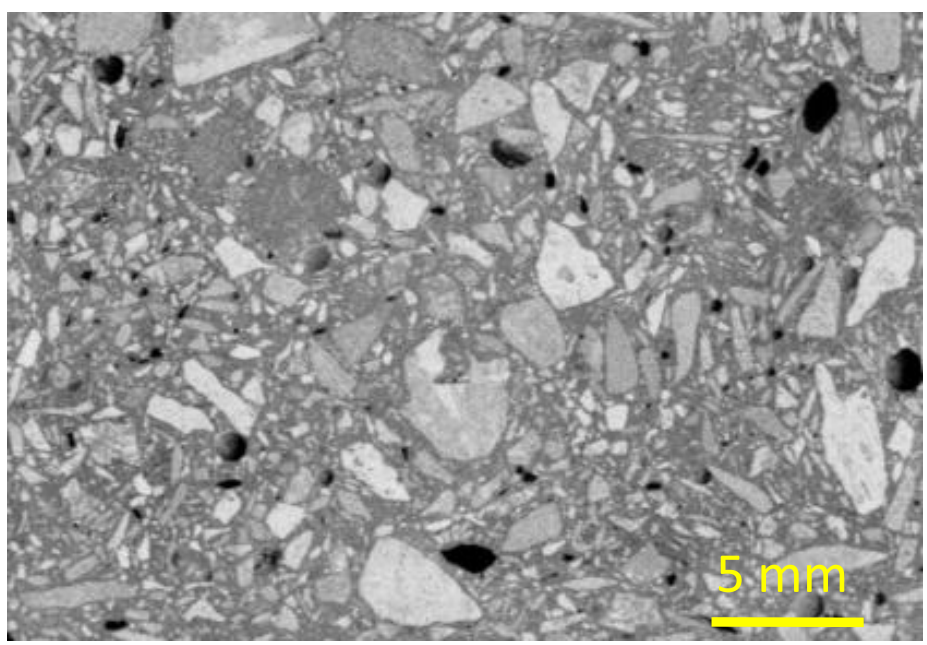

Figure 2: Calcium x-ray image. After grinding, the newly exposed surface was imaged in the $\mu$ XRF. Elemental images were derived from the hyperspectral data cube.

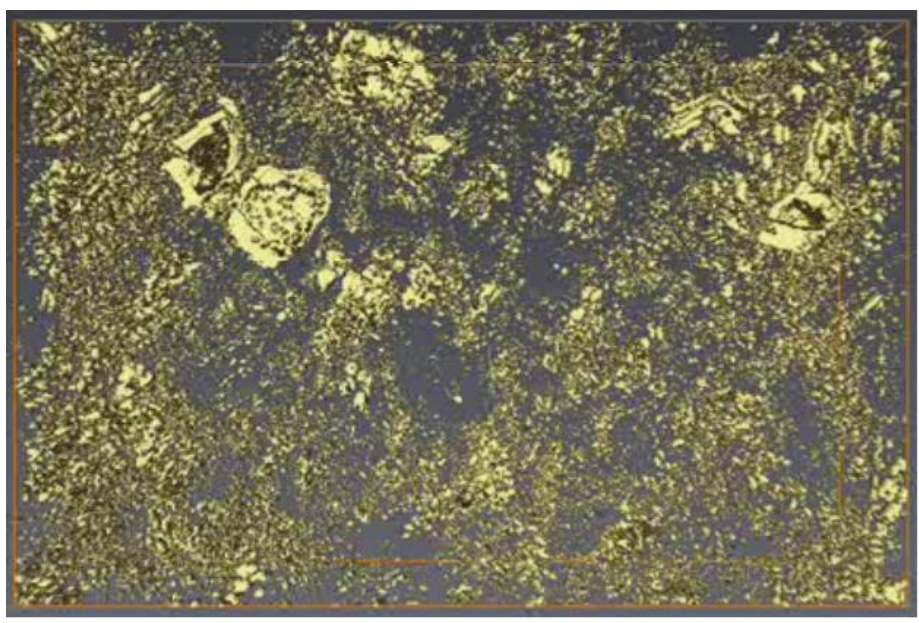

Figure 4: Derived single phase voxel map. Each phase was assembled into a 3 dimensional image to track the migration of each phase with depth. 